\title{
3 Research Square

\section{Notch1 protectives against ischemic reperfusion injury via suppressing PTEN-Pink1 mediated mitochondrial dysfunction and mitophagy}

\section{Qi-rong Xu}

First Affiliated Hospital of Nanchang University

Sheng Liu

First Affiliated Hospital of Nanchang University

Qiang Gong

First Affiliated Hospital of Nanchang University

\section{Rong-rong Zhu}

Gaoxin Hospital of the First Affiliated Hospital of Nanchang University

Ji-chun Liu

First Affiliated Hospital of Nanchang University

Xue-liang Zhou ( $\boldsymbol{D}$ dr_zhouxliang@163.com)

Nanchang University https://orcid.org/0000-0003-2879-2882

\section{Research Article}

Keywords: Notch1, PTEN, Pink1-Mfn2-Parkin, ischemic reperfusion injury

Posted Date: September 29th, 2022

DOI: https://doi.org/10.21203/rs.3.rs-1333462/v2

License: (c) (i) This work is licensed under a Creative Commons Attribution 4.0 International License. Read Full License 


\section{Abstract \\ Background}

Myocardial ischemia/reperfusion injury is associated with adverse cardiovascular outcomes after acute myocardial infarction. However, the molecular mechanism of ischemia/reperfusion injury remains unclear. Mitochondria dysfunction have become important participants and regulators of myocardial ischemia-reperfusion injury. However, the molecular mechanisms involved in this process are still not fully understood. We previously reported that Notch1 can reduce mitochondrial lysis, reduce myocardial infarct size and inhibit ventricular remodeling. Herein, we tried to explore the downstream target of Notch1 on the mitochondrial regulation.

\section{Methods}

Ischemic/reperfusion injury rat model and hypoxia/reoxygenation cell model were constructed in this study. The expression of PTEN was detected by real-time PCR, Western Blot and immunofluorescence staining. The cell viability was analyzed by CCK-8. The apoptosis level was detected by TUNEL assay. The mitochondrial fission/fusion were analyzed by Mito-Tracker Green staining. Measurement of cardiac troponin I (cTnl), lactate dehydrogenase (LDH), superoxide dismutase (SOD) and CK levels creatine kinase-MB (CK) were detected by ELISA Kits.

\section{Results}

We found that PETN-Pink1-Parkin signaling is inhibited by Notch1 I/R injured neonatal cardiomyocytes and hearts, thus via inhibiting the mitochondrial dysfunction and fragmentation. With the recure of PTEN or Pink1, the protective effect of Notch1 was largely diminished.

\section{Conclusion}

These results suggest that N1ICD protective against ischemic reperfusion injury via suppressing PTENPink1 mediated mitochondrial dysfunction and fragmentation.

\section{Introduction}

Reperfusion is the only way to salvage ischemic myocardial from infarction, but reperfusion itself can cause additional damage, so the resulting myocardial infarction size is determined by both the ischemia and reperfusion injury. Myocardial ischemia/reperfusion injury $(I / R)$ is a major health problem in China, and its morbidity and mortality are increasing year by year ${ }^{[1-4]}$. The development of acute heart failure is the leading cause of death after acute myocardial ischemia/reperfusion injury. Advances in medical 
technology, such as medical thrombolysis, interventional stents, and bypass surgery, have significantly reduced mortality in patients with acute myocardial I/R, and thus experience acute heart failure $\mathrm{e}^{[4-10]}$. Promoting myocardial I/R region repair is the key to prevent heart failure and reduce long-term mortality after MI /R.

The neurogenic locus notch homolog protein (Notch) signaling pathway plays a role in regulating cardiac development and the proliferation and differentiation of cardiomyocytes ${ }^{[11]}$. The Notch1 intracytoplasmic domain (NICD) translocate into the nucleus and regulates Hes 1 gene expression by binding to ubiquitous transcription factors and centromere-binding protein 1. Studies have shown that Notch signaling pathway plays an important role in the genesis, development and pathophysiology of the cardiovascular system. The role of Notch1 receptor and its downstream signaling molecule Hes 1 in ischemic heart disease was studied. Activation of Notch1 during myocardial I/R may activate downstream Hes 1 molecules, thereby inhibiting the expression of phosphatase and tensin homologues, thereby mitigating myocardial I/R damage. Activation of myocardial Notch1 signaling pathway protects ischemic myocardium by activating downstream Hes1 signaling and alleviating MI/R injury induced oxidative stress and nitrate stress injury. Akt signaling pathway plays a key role in cell survival, growth and migration. It may also be a survival signal during myocardial ischemia, and activation of this signal can exert an anti-apoptotic effect through upstream and downstream signaling pathways ${ }^{[12-15]}$. We previously reported that Notch1 regulates the dynamic balance between mitochondrial fusion/division and mitochondrial autophagy in I/R injury cardiomyocytes, and provides protection for cardiomyocytes ${ }^{[16,17]}$. It is reported that Notch1 is associated with the regulation of PTEN through recruitment of C-promoter-binding factor-1 (CBF-1), a Notch transcription factor, and binding to the PTEN promoter. On the other hand, PTEN-Pink1-MFN2-Parkin signaling pathway largely involved in the progress of myocardial ischemia/reperfusion injury. However, the regulatory mechanism of Notch1 signaling pathway on the PTEN/Akt signaling signaling pathway and its actual role on I/R injury are still unclear.

Herein, we demonstrate that Notch1 impaired the elevated expression of PETN in I/R injured heart and cardiomyocytes. Moreover, we found that PTEN over-expression impaired the protective effect of N1 ICD against hypoxia/reoxygenation induced injury, mitochondrial fragmentation and mitophagy in neonatal cardiomyocytes and hearts. These results suggest that Notch1 regulated PTEN-Pink1-MFN2-Parkin signaling might become a promising therapeutic strategy for treating myocardial I/R injury.

\section{Materials And Methods}

\subsection{Animal care}

Animals used in this study were maintained in accordance with the Guide for the Care and Use of Laboratory Animals (Publication $85-23$, National Institutes of Health, Bethesda, MD), and all procedures were approved by the First Affiliated Hospital of Nanchang University (Nanchang, Jiangxi, China). 


\subsection{Isolation and culture of rat neonatal ventricular myocytes}

Rat neonatal left ventricular myocytes were isolated from adult rat hearts using a standard enzymatic method as previously described ${ }^{[17]}$.

\subsection{Simulated H/R in isolated cardiomyocytes}

A cellular model of simulated $\mathrm{H} / \mathrm{R}$ (20-min/30-min) in neonatal left ventricular myocytes was used as previously described ${ }^{[18]}$. Briefly, the primary neonatal left ventricular myocytes were cultured in anoxic solution in an anoxic incubator $\left(95 \% \mathrm{~N}_{2} / 5 \% \mathrm{CO}_{2}\right)$ for 3 hours. The hypoxia solution was then replaced by reoxygenation solution and cultured in a high-oxygen incubator $\left(95 \% \mathrm{O}_{2} / 5 \%-\mathrm{CO}_{2}\right)$ for 3 hours.

\subsection{Construction and infection of recombinant adenoviruses}

Recombinant adenoviruses expressing Rat N1ICD/PTEN/Pink1 complementary DNA (cDNA) were prepared using the pAdEasyTM vector system (Qbiogene) as described previously. Primary cardiac myocytes were infected with adenoviral particles at the multiplicity of infection of 100 .

\subsection{Cell viability assay}

The cell viability of neonatal cardiomyocytes was detected with CCK-8 assay (Dojindo) as described previously ${ }^{[18]}$.

\subsection{Real-time PCR}

Total RNA was extracted from frozen heart tissues or cultured cells and RNA reverse-transcription were performed as we previously described. RT-PCR was conducted using a SYBR Green Master Mix (Cowin, Beijing, China). All the experiments were repeated three times independently.

\subsection{Western blot analysis}

The adult cardiomyocytes were lysed in cell lysis buffer (Beyotime Institute of Biotechnology) at $4^{\circ} \mathrm{C}$. Protein samples were separated by $8 \%-10 \%$ SDS-PAGE, then transferred to nitrocellulose membranes (Millipore). Membranes were incubated with primary antibodies overnight at $4^{\circ} \mathrm{C}$, followed by incubation with secondary antibodies at room temperature for 1 hour. The fluorescent signals were detected using enhanced chemiluminescence by ImageQuant LAS4000 (GE). All the experiments were repeated three times independently.

\subsection{Immunofluorescence analysis}

Immunofluorescence assay was carried out as described above. The muscle cells fixed with paraformaldehyde were treated with $0.5 \%$ Triton X-100 PBS for 15 minutes. The myocytes were then immunostained with anti-PTEN (1:100) antibody. After washing with PBS, FITC-conjugated anti-rabbit IgG 
(1:2000, Jackson, USA) were stained for $2 \mathrm{~h}$, respectively. The stained cells were observed under a Zeiss LSM800 confocal microscope (Zeiss, Heidelberg, Germany).

\subsection{Hes1 and PTEN promoter luciferase reporter assay}

Commercial PTEN luciferase reporter kit from SABiosciences Qiagen was used to determine the effect of HES1 over-expression on transcriptional activity of PTEN. Neonatal cardiomyocytes cells were transfected with the reporter construct using Lipofectamine 3000 (Thermo Fisher). Twenty-four hours after transfection, cells were harvested using lysis buffer. Samples were centrifuged, and $20 \mathrm{~mL}$ aliquot was used for measurement of dual luciferase activity using a luminometer.

\subsection{TUNEL staining}

Apoptosis rates in cultured neonatal cardiomyocytes and mouse heart tissue sections were analyzed by TUNEL staining using the in-situ cell death detection kit (Roche Applied Science) according to the manufacturer's protocol. In summary, the slides were incubated with TUNEL reaction mixture, apoptotic cells were labeled, and the total number of cells was determined by DAPI staining. The slides were viewed under a Zeiss LSM800 laser scanning confocal microscope (Zeiss, Wetzlar, Germany). Apoptosis rate was calculated as the percentage of TUNEL positive cells in the total number of DAPI stained cells. All the experiments were repeated three times independently.

\subsection{Measurement of cTnl, lactate dehydrogenase (LDH), superoxide dismutase (SOD) and CK levels creatine kinase-MB (CK)}

The coronary effluent and culture medium of cardiomyocytes after reperfusion were placed in a thermostatic chamber. Electrochemiluminescence immunoassay was used to detect the level of cTnl, LDH and CK according to the instructions of the kit (Roche, Germany). The measurement of SOD, activity is performed according to the test kit instructions (Nanjing Kaiji Bio, Nanjing, China). All the experiments were repeated three times independently.

\subsection{Seahorse analysis}

As previously described, mitochondrial metabolic flux was tested in adult cardiomyocytes. Briefly, cardiomyocytes were inoculated into XF24 hippocampal plate coated with laminin at a density of 104 cells per well and cultured overnight in BCAA-free substrate-restricted medium, then analyzed. OCR and ECAR were determined using the Seahorse XF24 extracellular flux analyzer (Seahorse Bioscience, North Billerica, MA, USA). All the experiments were repeated three times independently.

\subsection{Mitochondrial fusion/fission detection}

The mitochondrial fusion/fission detection of myocardiocytes was detected as described previously ${ }^{[19]}$.

\subsection{I/R injury model in Langendorff-perfused rat hearts}


I/R injury model in Langendorff-perfused rat hearts was performed as previously ${ }^{[19]}$. In brief, the rats were anesthetized with sodium pentobarbital ( $45 \mathrm{mg} / \mathrm{kg}$ I.P.), the hearts were rapidly resected-and the KrebsHenselite $(\mathrm{K}-\mathrm{H})$ solution was infused at $37^{\circ} \mathrm{C}$ using a Langendorff apparatus at a constant pressure of $80 \mathrm{~mm} \mathrm{Hg}$ as described in the previous paper. A water-filled latex balloon was connected to a pressure sensor (Gould P23DB, AD instrument) and inserted into the left ventricular cavity to achieve a stable LVEDP of $5-10 \mathrm{mmHg}$ during the initial equilibrium. After balanced perfusion, the heart was ischemia-free for 30 minutes, followed by another 45 minutes of perfusion. LVDP and $\pm \mathrm{dp} / \mathrm{dt}$ Max were evaluated using PowerLab system (AD instrument).

\subsection{In vivo adenoviral gene delivery}

The surgical procedures and adenoviral delivery were carried out as described ${ }^{[19]}$.

\subsection{Statistical analysis}

Data are expressed as mean \pm SEM. Statistical significance was determined by multiple comparisons or repeated measures using analysis of variance or repeated analysis of variance. The student $t$ test was used to estimate the significant difference between the two averages. $\mathrm{P}<0.05$ was considered statistically significant.

\section{Results}

\subsection{N1ICD impaired the hypoxia/reoxygenation elevated expression of PETN in neonatal cardiomyocytes.}

To illustrate the effect of N1ICD on the expression level of PTEN in H/R injured neonatal cardiomyocytes, we overexpressed N1ICD in the sham or H/R injured neonatal cardiomyocytes by adenovirus. The level of PTEN mRNA (Fig. 1A) and protein (Fig. 1B) were significantly increased in H/R group, whereas N1 ICE overexpression dramatically decreased this increasing. This decreasing was further confirmed by immunofluorescence staining (Fig. 1C). Considering Hes1 is a critical transcript factor of Notch1 signaling, we then test whether Hes1 transcriptionally regulate the expression of PTEN. RT-PCR (Fig. 1D) and real-time PCR (Fig. 1E) of Chromatin Immunoprecipitation (ChIP) assay showed that the promoter of PTEN were obviously enriched in the component of HES1 IP products. We then predicted the binding site of Hes1 on the promoter of PTEN with JASPAR tool (http://jaspar.genereg.net/) and constructed a luciferase promoter activity reporter with wildtype PTEN or mutant promoter sequence (Fig. 1D). The luciferase result showed that the transcriptional activity of PTEN was inhibited by Hes 1 over-expression (Fig. 1F). Moreover, dual-luciferase assay of the activity of PTEN promoter suggested that Hes1 significantly decreased the activity of wild-type PTEN promoter, but not the binding site mutant NRF2 promoter (Fig. 1F). These results demonstrate that N1ICD impaired the hypoxia/reoxygenation elevated expression of PETN in neonatal cardiomyocytes. 


\subsection{PTEN over-expression impaired the protective effect of N1ICD against hypoxia/reoxygenation induced injury in neonatal cardiomyocytes.}

To explore whether the N1ICD decreased PTEN expression contributed to the cardio protection effect of N1 ICD, we overexpressed N1ICD with or without PTEN in the H/R injured neonatal cardiomyocytes by adenovirus. Notch1 improved the cell viability of H/R injured neonatal cardiomyocytes (Fig. 2A). PETN did not affect the cell viability, whereas obviously decreased the cell viability in Notch1 over-expressed $\mathrm{H} / \mathrm{R}$ neonatal cardiomyocytes (Fig. 2A). Considering apoptosis is the major pathophysiological process underlying liver ischemia/reperfusion (I/R) injury. We then analyzed the $H / R$ induced apoptosis by TUNEL staining, the percentage of $H / R$ induced apoptotic cardiomyocytes was decreased by Notch1 overexpression (Fig. 2B), which was dramatically elevated by PTEN over-expression. As cTnl is $100 \%$ tissuespecific for myocardial damage and is an excellent marker for myocardial injury, the cardiac troponin I (cTnl) levels in the culture medium were also detected at the end of the reperfusion to assess myocardial injury. The results showed that the Notch1 over-expression impaired cTnl level was dramatically elevated by PTEN over-expression (Fig. 2C). Consistently, the activity of LDH (Fig. 2D) and CK (Fig. 2E) that were also used as indicators of myocardial injury were markedly decreased following H/R in Notch1 overexpression group. However, PTEN over-expression could markedly reversed these effects induced by H/R. Concurrently, the activity of the antioxidative cytokines SOD was evaluated in Notch1 over-expression group, but not in the PTEN over-expression group (Fig. 2F). These results demonstrate that PTEN overexpression impaired the protective effect of N1ICD against hypoxia/reoxygenation induced injury in neonatal cardiomyocytes.

\subsection{PTEN over-expression impaired the protective effect of N1ICD against hypoxia/reoxygenation induced mitochondrial dysfunction and fragmentation in neonatal cardiomyocytes.}

To further verified Notch1 protected mitochondrial respiration in H/R injured neonatal cardiomyocytes via suppressing PTEN, we then analyzed the mitochondrial function and respiration in neonatal cardiomyocytes with Notch1 with or without PTEN over-expression. We found that the ATP content (Fig. 3A) and ATP synthase activity (Fig. 3B) were significantly increased by Notch1 over-expression. PTEN over-expression dramatically impaired the ATP content and ATP content (Fig. 3A) and ATP synthase activity (Fig. 3B). Furthermore, the oxygen consumption rate of neonatal cardiomyocytes, including maximal respiration (MR) and spare respiratory capacity (SPR), increased by Notch1 overexpression, whereas decreased by PTEN over-expression (Fig. 3C). Consistently, the H/R induced mitochondrial fragmentation, indicated by the fragmented mitochondrial numbers was also decreased by Notch1 over-expression, which was reversed by PTEN over-expression (Fig. 4A). Furthermore, the level of mitochondrial fragmentation markers, including Pink1, phosphorylated MFN2 and phosphorylated Parkin, were inhibited by Notch1 over-expression, but reversed by PTEN over-expression (Fig. 4B). As a process that selectively removes damaged organelles by autolysosome degradation, mitophagy is an early cellular response to ischemia in cardiomyocytes during I/R and cardiac ischemic diseases. In our results, 
Notch1 obviously inhibited the H/R induced mitophagy in neonatal cardiomyocytes (Fig. 4C).

Mitochondrial fragmentation in apoptosis mitochondrial outer membrane permeabilization is associated with pro-apoptotic stimuli, which results in the release of cytochrome $c$ from the mitochondria. We also found that Notch1 over-expression significantly decreased the cytoplastic cytochrome c, whereas PTEN over-expression elevated it (Fig. 4D). Collectively, PTEN over-expression impaired the protective effect of N1ICD against hypoxia/reoxygenation induced mitochondrial dysfunction and fragmentation in neonatal cardiomyocytes.

\subsection{Pink1 over-expression impaired the protective effect of N1ICD against hypoxia/reoxygenation induced injury in neonatal cardiomyocytes.}

Pink1 on the outer membranes of impaired mitochondria promotes mitophagy and regulates mitochondrial morphology. To verified whether Pink1 signaling is critical to the PTEN impaired cardio protection effect of Notch1, we overexpressed N1 ICD with or without Pink1 over-expression in the H/R injured neonatal cardiomyocytes by adenovirus. Similarly, Notch1 improved the cell viability of $\mathrm{H} / \mathrm{R}$ injured neonatal cardiomyocytes (Fig. 5A) and Pink1 did not affect the cell viability, whereas Pink1 overexpression obviously decreased the cell viability in Notch1 over-expressed H/R neonatal cardiomyocytes (Fig. 5A). The percentage of Notch1 decreased apoptosis in H/R neonatal cardiomyocytes was increased by Pink1 over-expression (Fig. 5B). Moreover, the Notch1 decreased markers for myocardial injury, including cTnl, LDH activity (Fig. 5C) and CK activity (Fig. 5C) were markedly increased following H/R in Pink1 over-expression group. These results demonstrate that Pink1 over-expression impaired the protective effect of N1ICD against hypoxia/reoxygenation induced injury in neonatal cardiomyocytes.

3.5 Pink1 over-expression impaired the protective effect of N1ICD against hypoxia/reoxygenation induced mitochondrial dysfunction and fragmentation in neonatal cardiomyocytes

Similar with the PTEN over-expression, we found that the Notch1 preserved ATP content (Fig. 6A) and ATP synthase activity (Fig. 6B) in H/R neonatal cardiomyocytes were significantly decreased by Pink1 over-expression. Furthermore, the Notch1 preserved oxygen consumption rate of neonatal cardiomyocytes, including maximal respiration (MR) and spare respiratory capacity (SPR), obviously decreased by Pink1 over-expression (Fig. 6C). Consistently, the Notch1 decreased mitochondrial fragmentation (Fig. 7A) and mitophagy (Fig. 7B) was also increased by Pink1 over-expression. These results demonstrates that Pink1 over-expression impaired the protective effect of N1ICD against hypoxia/reoxygenation induced mitochondrial dysfunction and fragmentation in neonatal cardiomyocytes.

3.7 N1 ICD protective against ischemic reperfusion injury via suppressing PTEN-Pink1 mediated mitochondrial dysfunction and fragmentation in vivo 
To confirm the critical role of suppressed PTEN-Pink1 signaling in the cardioprotective effect of Notch1 in vivo, as shown in Fig. 8A, we over-expressed Notch1 with or without PTEN/Pink1 and analyzed the postischemic contractile function in Langendorff-perfused rat hearts. The preischemic contractile parameters were similar between the five groups, while the I/R (30 min/45 min)-suppressed LV contractile function, characterized by LV develpment pressure (LVDP), LV end-diastolic pressure (LVEDP), and maximal speed of LV pressure development and decline $( \pm \mathrm{dp} / \mathrm{dt})$, was markedly alleviated by Notch1 over-expression (Fig. 8A). However, with the over-expression of PTEN or Pink1, the cardioprotective effect of Notch1 on the contractile function were totally abrogated (Fig. 8A). Consistently, I/R-induced cTnl level (Fig. 8B) and LDH activity (Fig. 8C) in coronary perfusate were significantly inhibited by Notch1 overexpression, whereas dramatically impaired by the over-expression of PTEN or Pink1 respectively. Moreover, the apoptotic percentage of myocardium was determined by TUNEL assay and the results indicated that the the positive staining of TUNEL in the Notch1 groups was significantly decreased, whereas obviously increased in PTEN or Pink1 over-expression hearts (Fig. 8C). Similar with the in vitro results, the level of mitochondrial fragmentation markers, including Pink1, phosphorylated MFN2 and phosphorylated Parkin, were inhibited by Notch1 over-expression, but reversed by PTEN over-expression (Fig. 8D). Furthermore, Notch1 over-expression significantly decreased the cytoplastic cytochrome c, whereas PTEN or Pink1 over-expression elevated it (Fig. 8E). Collectively, PTEN over-expression impaired the protective effect of N1ICD against hypoxia/reoxygenation induced mitochondrial dysfunction and fragmentation in neonatal cardiomyocytes. Collectively, these results demonstrates that N1 ICD protective against ischemic reperfusion injury via suppressing PTEN-Pink1 mediated mitochondrial dysfunction and fragmentation in vivo.

\section{Discussion}

Mitochondria have become important participants and regulators of myocardial ischemia-reperfusion injury ${ }^{[20-25]}$. Emerging studies showed that Notch1 protects the heart from I/R damage, but the underlying mechanism is not fully understood. We previously reported that Notch1 activates RISK/SAFE/HIF-1 alpha signal, reduces ROS in cardiomyocytes, enhances cardiomyocyte viability, improves mitochondrial fusion, and significantly reduces myocardial I/R injury ${ }^{[16-19]}$. In this study, we demonstrate that Notch1 preserved the mitochondrial respiration and inhibited mitochondrial fragmentation via suppressing Pink1-Parkin signaling pathway in I/R injured neonatal cardiomyocytes and heart.

The Notch signaling pathway is a conserved pathway guided by ligand-dependent processes that ultimately release the Notch intracellular domain (NIC) from its membrane progenitor cells, allowing translocation to the nucleus to initiate transcription ${ }^{[26-29]}$. In the heart, Notch is expressed in a variety of cell types, including cardiomyocytes, smooth muscle cells, and endothelial cells ${ }^{[30]}$. We have previously found that Notch1 signal is activated in the process of myocardial ischemic pre-condition and ischemic post-condition, which can improve cell viability and inhibit apoptosis ${ }^{[16]}$. In addition, the activated Notch1 signal stabilized the mitochondrial membrane potential and reduced IRI induced reactive oxygen species. 
In addition, in the Langendorff cardiac perfusion model, activated Notch1 signal restores cardiac function, reduces lactate dehydrogenase release, and limits infarct size after myocardial ischemia ${ }^{[16]}$. We also reported that Notch1 can reduce mitochondrial lysis, reduce myocardial infarct size and inhibit ventricular remodeling. However, the downstream target of Notch1 on the mitochondrial regulation remains unclear. Herein, we further found that N1ICD protective against ischemic reperfusion injury via suppressing PTEN-Pink1 mediated mitochondrial dysfunction and fragmentation.

Pink1 has a total length of 581 amino acids, including an N-terminal mitochondrial targeting sequence, a transmembrane domain, a highly conserved serine/threonine kinase domain, and a C-terminal autoregulatory domain. Under physiological conditions, due to rapid degradation of PINK1, the level of PINK1 was low ${ }^{[31-33]}$. Pink1 acts upstream of Parkin and is necessary for Parkin to activate and recruit depolarized mitochondria ${ }^{[34,35]}$. Pink1 and Parkin have been shown to regulate many different mitochondria related activities, such as biogenesis, integrity, respiration, fission, and fusion, in addition to their roles in the execution of autophagy ${ }^{[36-39]}$. In this study, we showed that Pink1 over-expression impaired the protective effect of N1ICD against hypoxia/reoxygenation induced injury in neonatal cardiomyocytes. Moreover, Pink1 over-expression impaired the protective effect of N1ICD against hypoxia/reoxygenation induced mitochondrial dysfunction and fragmentation in neonatal cardiomyocytes. In the in vivo data, we verified that N1ICD protective against ischemic reperfusion injury via suppressing PTEN-Pink1 mediated mitochondrial dysfunction and fragmentation.

In conclusion, this study presented a detailed mechanism by which Notch1 impairs mitochondrial dysfunction and fragmentation vis suppressing PTEN-Pink1-Pakin signaling during myocardial protection. Further studies are needed to determine the clinical significance of these effects and to develop new therapies for patients with $\mathrm{I} / \mathrm{R}$, as treating this disease remains clinically challenging.

\section{Declarations}

\section{Funding}

This work was supported by grants from the National Natural Science Foundation of China (No. $82070303,81970199,81860045$, and 81860054), the Natural Science Foundation of Jiangxi Province (No. 20192ACB21030, 20192ACBL20036, 20181BAB205003) and the innovation of science and technology in Jiangxi Province outstanding young talent training plan (No. 20192BCBL23022).

\section{Ethics approval}

The procedures of animal experiments were approved by the First Affiliated Hospital of Nanchang University (Nanchang, Jiangxi, China).

\section{Consent for publication}

All authors have informed and consented to the publication of this article 


\section{Conflicts}

The authors declare no competing interests regarding this manuscript.

\section{Availability of data and material}

The data in this study are available from the corresponding author on reasonable request.

\section{Authors' contributions}

Qi-rong Xu, Sheng Liu and Qiang Qong performed all experiments. Ji-chun Liu and Xue-liang Zhou designed this study. Rong-rong Zhu analyzed a part of the data. Xue-liang Zhou drafted the manuscript. All authors read and approved the final manuscript.

\section{Acknowledgements}

A preprint has previously been published ${ }^{[40]}$.

\section{References}

1. Heusch G. Myocardial ischaemia-reperfusion injury and cardioprotection in perspective. Nat Rev Cardiol. 2020; 17 (12):773-789.

2. Jennings RB, Steenbergen C, Jr., Reimer KA. Myocardial ischemia and reperfusion. Monogr Pathol. 1995; 37:47-80.

3. Michael LH, Entman ML, Hartley CJ, Youker KA, Zhu J, Hall SR, Hawkins HK, Berens K, Ballantyne CM. Myocardial ischemia and reperfusion: a murine model. Am J Physiol. 1995; 269 (6 Pt 2):H21472154.

4. Robson A. A novel cardioprotective function for DRP1 inhibition. Nat Rev Cardiol. 2021; 18 (5):306307.

5. Heusch G, Rassaf T. Left Ventricular Unloading in Myocardial Infarction: Gentle Reperfusion Through the Backdoor? J Am Coll Cardiol. 2020; 76 (6):700-702.

6. Hinkel R, Ramanujam D, Kaczmarek V, Howe A, Klett K, Beck C, Dueck A, Thum T, Laugwitz KL, Maegdefessel L, Weber C, Kupatt C, Engelhardt S. AntimiR-21 Prevents Myocardial Dysfunction in a Pig Model of Ischemia/Reperfusion Injury. J Am Coll Cardiol. 2020; 75 (15):1788-1800.

7. Stevens RM, Jahania MS, Stivers JE, Mentzer RM, Jr., Lasley RD. Effects of in vivo myocardial ischemia and reperfusion on interstitial nitric oxide metabolites. Ann Thorac Surg. 2002; 73 (4):12611266.

8. Swain L, Reyelt L, Bhave S, Qiao X, Thomas CJ, Zweck E, Crowley P, Boggins C, Esposito M, Chin M, Karas RH, O'Neill W, Kapur NK. Transvalvular Ventricular Unloading Before Reperfusion in Acute Myocardial Infarction. J Am Coll Cardiol. 2020; 76 (6):684-699. 
9. Tibaut M, Mekis D, Petrovic D. Pathophysiology of Myocardial Infarction and Acute Management Strategies. Cardiovasc Hematol Agents Med Chem. 2017; 14 (3):150-159.

10. Binder A, Ali A, Chawla R, Aziz HA, Abbate A, Jovin IS. Myocardial protection from ischemiareperfusion injury post coronary revascularization. Expert Rev Cardiovasc Ther. 2015; 13 (9):10451057.

11. Penton AL, Leonard LD, Spinner NB. Notch signaling in human development and disease. Semin Cell Dev Biol. 2012; 23 (4):450-457.

12. Dabral S, Tian X, Kojonazarov B, Savai R, Ghofrani HA, Weissmann N, Florio M, Sun J, Jonigk D, Maegel L, Grimminger F, Seeger W, Savai Pullamsetti S, Schermuly RT. Notch1 signalling regulates endothelial proliferation and apoptosis in pulmonary arterial hypertension. Eur Respir J. 2016; 48 (4):1137-1149.

13. Del Monte-Nieto G, Ramialison M, Adam AAS, Wu B, Aharonov A, D'Uva G, Bourke LM, Pitulescu ME, Chen H, de la Pompa JL, Shou W, Adams RH, Harten SK, Tzahor E, Zhou B, Harvey RP. Control of cardiac jelly dynamics by NOTCH1 and NRG1 defines the building plan for trabeculation. Nature. 2018; 557 (7705):439-445.

14. Rocca C, Femmino S, Aquila G, Granieri MC, De Francesco EM, Pasqua T, Rigiracciolo DC, Fortini F, Cerra MC, Maggiolini M, Pagliaro P, Rizzo P, Angelone T, Penna C. Notch1 Mediates Preconditioning Protection Induced by GPER in Normotensive and Hypertensive Female Rat Hearts. Front Physiol. 2018; 9:521.

15. Si X, Zheng H, Wei G, Li M, Li W, Wang H, Guo H, Sun J, Li C, Zhong S, Liao W, Liao Y, Huang S, Bin J. circRNA Hipk3 Induces Cardiac Regeneration after Myocardial Infarction in Mice by Binding to Notch1 and miR-133a. Mol Ther Nucleic Acids. 2020; 21:636-655.

16. Zhou XL, Wan L, Xu QR, Zhao Y, Liu JC. Notch signaling activation contributes to cardioprotection provided by ischemic preconditioning and postconditioning. $J$ Trans/ Med. 2013; 11:251.

17. Zhou XL, Wu X, Xu QR, Zhu RR, Xu H, Li YY, Liu S, Huang H, Xu X, Wan L, Wu QC, Liu JC. Notch1 provides myocardial protection by improving mitochondrial quality control. J Cell Physiol. 2019; 234 (7):11835-11841.

18. Zhou XL, Wu X, Zhu RR, Xu H, Li YY, Xu QR, Liu S, Lai SQ, Xu X, Wan L, Wu QC, Liu JC. Notch1-Nrf2 signaling crosstalk provides myocardial protection by reducing ROS formation. Biochem Cell Biol. 2020; 98 (2):106-111.

19. Dai SH, Wu QC, Zhu RR, Wan XM, Zhou XL. Notch1 protects against myocardial ischaemiareperfusion injury via regulating mitochondrial fusion and function. J Cell Mol Med. 2020; 24 (5):3183-3191.

20. Boengler K, Lochnit G, Schulz R. Mitochondria "THE" target of myocardial conditioning. Am J Physiol Heart Circ Physiol. 2018; 315 (5):H1215-H1231.

21. Bugger H, Pfeil K. Mitochondrial ROS in myocardial ischemia reperfusion and remodeling. Biochim Biophys Acta Mol Basis Dis. 2020; 1866 (7):165768. 
22. Di Lisa F, Canton M, Menabo R, Kaludercic N, Bernardi P. Mitochondria and cardioprotection. Heart Fail Rev. 2007; 12 (3-4):249-260.

23. Ertracht $\mathrm{O}$, Malka A, Atar S, Binah $\mathrm{O}$. The mitochondria as a target for cardioprotection in acute myocardial ischemia. Pharmacol Ther. 2014; 142 (1):33-40.

24. Lesnefsky EJ, Chen Q, Tandler B, Hoppel CL. Mitochondrial Dysfunction and Myocardial IschemiaReperfusion: Implications for Novel Therapies. Annu Rev Pharmacol Toxicol. 2017; 57:535-565.

25. Paradies G, Paradies V, Ruggiero FM, Petrosillo G. Mitochondrial bioenergetics and cardiolipin alterations in myocardial ischemia-reperfusion injury: implications for pharmacological cardioprotection. Am J Physiol Heart Circ Physiol. 2018; 315 (5):H1341-H1352.

26. Adamowicz M, d'Adda di Fagagna F, Vermezovic J. NOTCH1 modulates activity of DNA-PKcs. Mutat Res. 2018; 808:20-27.

27. Zanotti S, Canalis E. Notch Signaling and the Skeleton. Endocr Rev. 2016; 37 (3):223-253.

28. Kim MY, Park JY, Park HS. Akt1-Mediated Phosphorylation of RBP-Jk Controls Notch1 Signaling. Biochemistry (Mosc). 2019; 84 (12):1537-1546.

29. Shah PA, Huang C, Li Q, Kazi SA, Byers LA, Wang J, Johnson FM, Frederick MJ. NOTCH1 Signaling in Head and Neck Squamous Cell Carcinoma. Cells. 2020; 9 (12).

30. Aquila G, Kostina A, Vieceli Dalla Sega F, Shlyakhto E, Kostareva A, Marracino L, Ferrari R, Rizzo P, Malaschicheva A. The Notch pathway: a novel therapeutic target for cardiovascular diseases? Expert Opin Ther Targets. 2019; 23 (8):695-710.

31. Barazzuol L, Giamogante F, Brini M, Cali T. PINK1/Parkin Mediated Mitophagy, Ca(2+) Signalling, and ER-Mitochondria Contacts in Parkinson's Disease. Int J Mol Sci. 2020; 21 (5).

32. Barodia SK, Creed RB, Goldberg MS. Parkin and PINK1 functions in oxidative stress and neurodegeneration. Brain Res Bull. 2017; 133:51-59.

33. Bueno M, Lai YC, Romero Y, Brands J, St Croix CM, Kamga C, Corey C, Herazo-Maya JD, Sembrat J, Lee JS, Duncan SR, Rojas M, Shiva S, Chu CT, Mora AL. PINK1 deficiency impairs mitochondrial homeostasis and promotes lung fibrosis. J Clin Invest. 2015; 125 (2):521-538.

34. Dombi E, Mortiboys H, Poulton J. Modulating Mitophagy in Mitochondrial Disease. Curr Med Chem. 2018; 25 (40):5597-5612.

35. Eiyama A, Okamoto K. PINK1/Parkin-mediated mitophagy in mammalian cells. Curr Opin Cell Biol. 2015; 33:95-101.

36. Ivankovic D, Chau KY, Schapira AH, Gegg ME. Mitochondrial and lysosomal biogenesis are activated following PINK1/parkin-mediated mitophagy. J Neurochem. 2016; 136 (2):388-402.

37. Soutar MPM, Kempthorne L, Annuario E, Luft C, Wray S, Ketteler R, Ludtmann MHR, Plun-Favreau H. FBS/BSA media concentration determines CCCP's ability to depolarize mitochondria and activate PINK1-PRKN mitophagy. Autophagy. 2019; 15 (11):2002-2011.

38. Wang N, Zhu P, Huang R, Wang C, Sun L, Lan B, He Y, Zhao H, Gao Y. PINK1: The guard of mitochondria. Life Sci. 2020; 259:118247. 
39. Youle RJ, Narendra DP. Mechanisms of mitophagy. Nat Rev Mol Cell Biol. 2011; 12 (1):9-14.

40. Xu Q-r, Liu S, Gong Q, Zhu R-r, Liu J-c, Zhou X-I. Notch1 protectives against ischemic reperfusion injury via suppressing PTEN-Pink1 mediated mitochondrial dysfunction and mitophagy. Research Square, 2022.

\section{Figures}

A

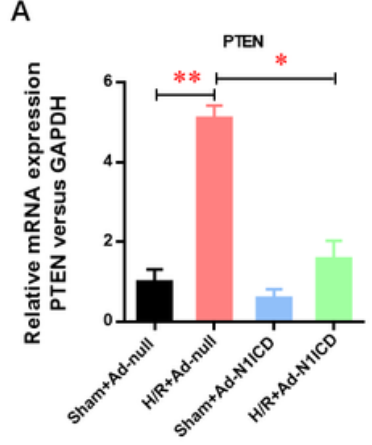

C
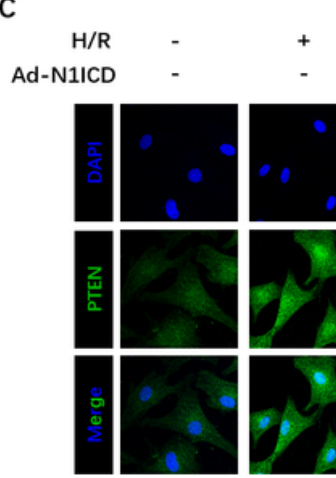

B

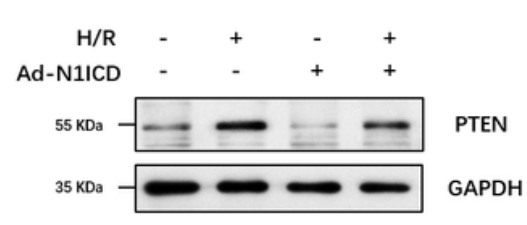

D

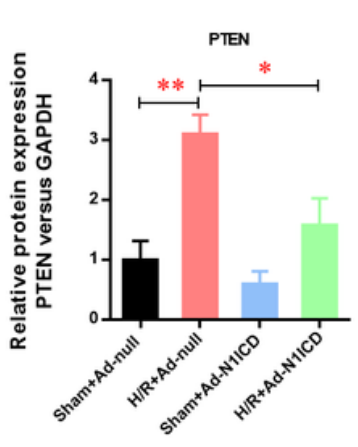

E

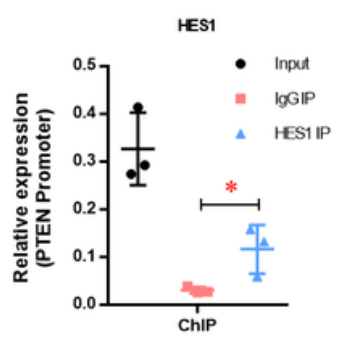

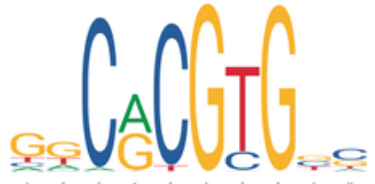

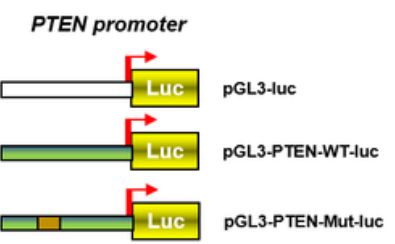

$\mathrm{F}$

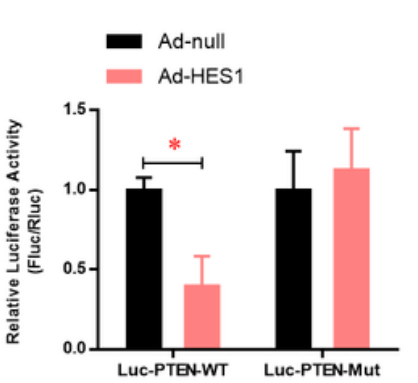

Figure 1

N1ICD impaired the hypoxia/reoxygenation elevated expression of PETN in neonatal cardiomyocytes. (A) The mRNA level of PTEN was analyzed by real-time PCR; (B) The protein level of PTEN was analyzed by real-time PCR; (C) The protein level of PTEN was analyzed by immunofluorescence staining assay; (D) ChIP-RT-PCR and (E) ChlpP-qPCR were performed to analyzed the enrichment of PTEN promoter in the ChIP products of Hes $1 ;(\mathrm{F})$ The promoter activity of PTEN was evaluated by luciferase assay; $\mathrm{N}=3$; ${ }^{2} P<05$ versus indicated group. 
A
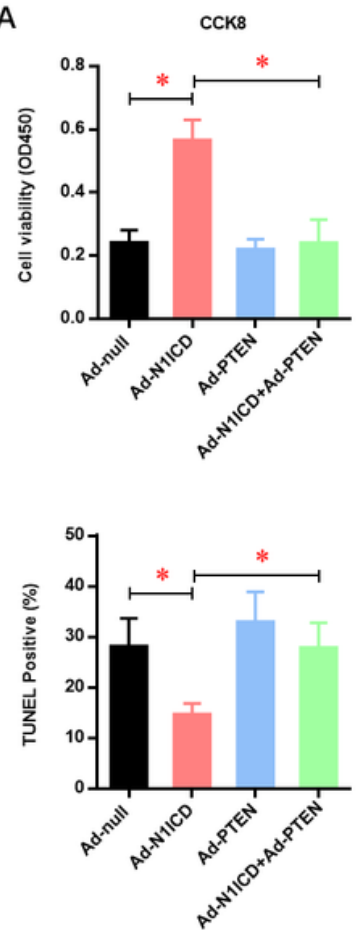

B
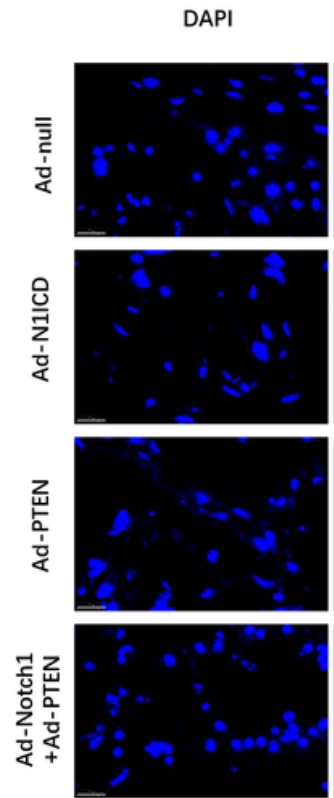

TUNEL
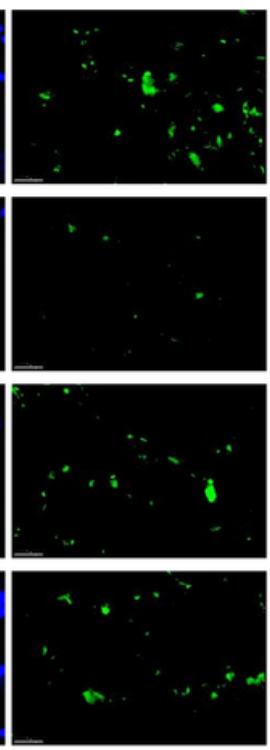

MERGE
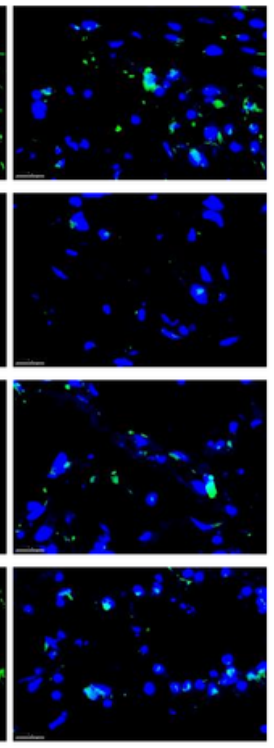

C
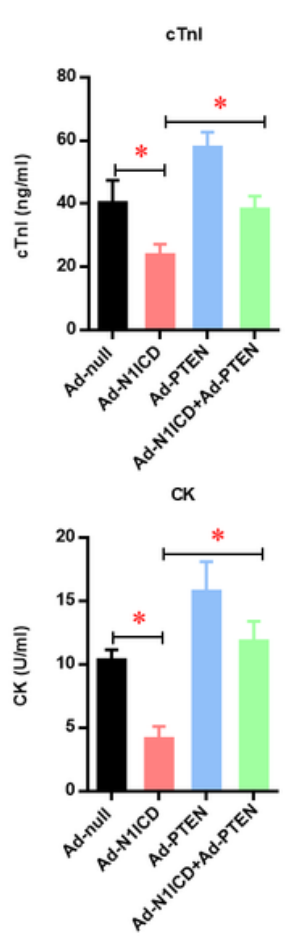

LH
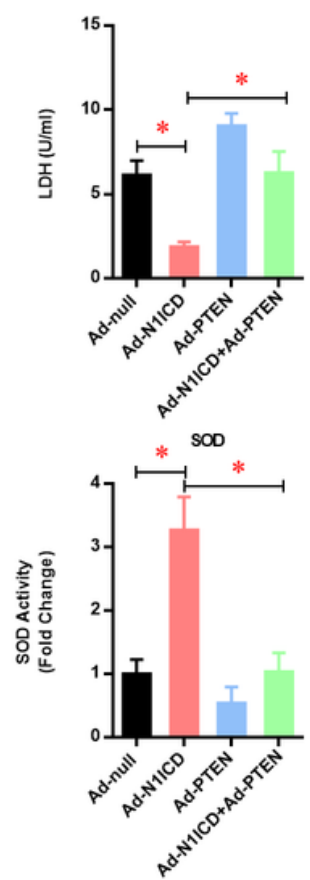

Figure 2

PTEN over-expression impaired the protective effect of N1ICD against hypoxia/reoxygenation induced injury in neonatal cardiomyocytes. (A) The cellular viability was detected by CCK-8 assay; (B) The percentage of apoptotic neonatal cardiomyocytes was analyzed by TUNE staining; (C) The level of cTnl in the culture medium were also detected at the end of the reperfusion to assess myocardial injury; (D) The activity of LDH and (E) CK were evaluated by Electrochemiluminescence immunoassay; (F) The levels of SOD were analyzed by ELISA Kit; $N=3$; ${ }^{*} P<05$ versus indicated group. 
A

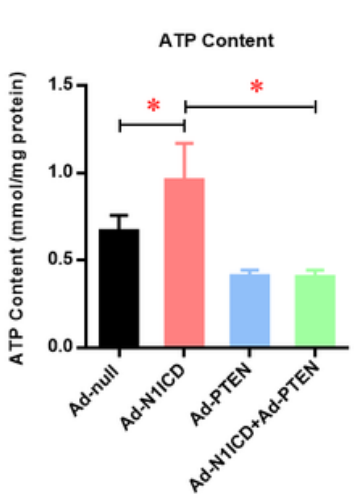

B

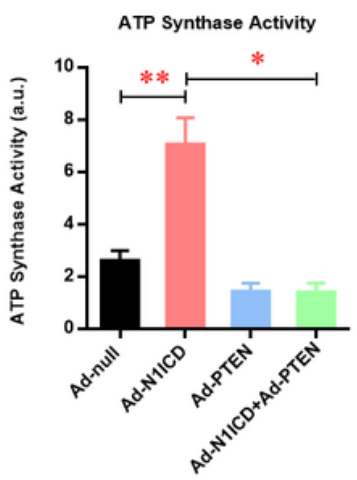

C
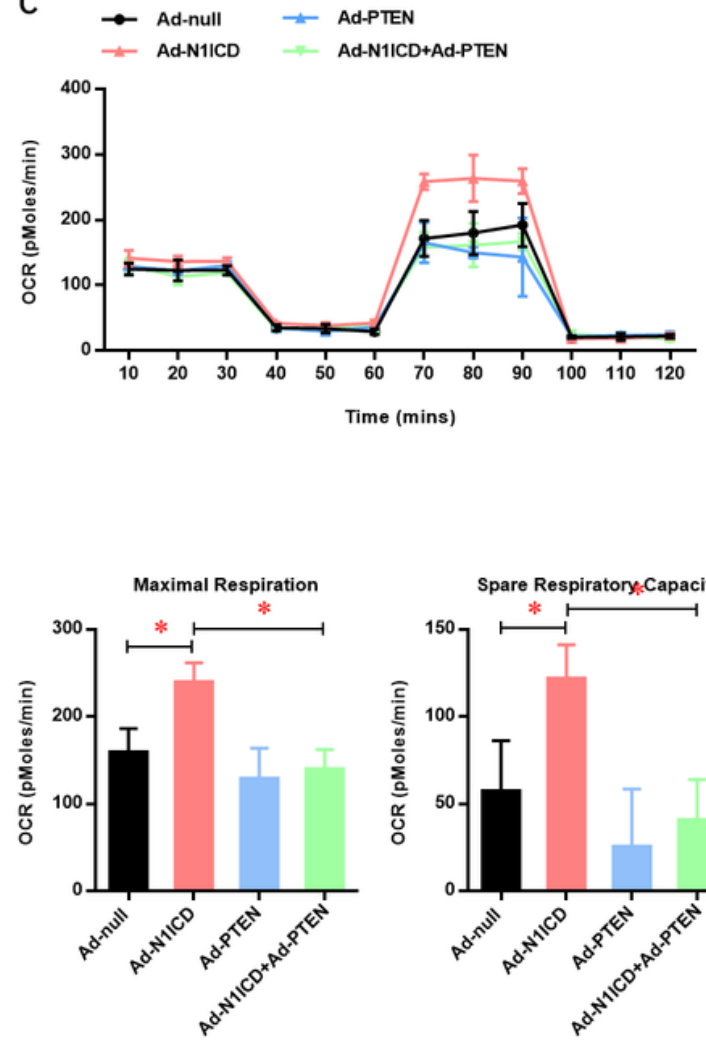

Basal Respiration
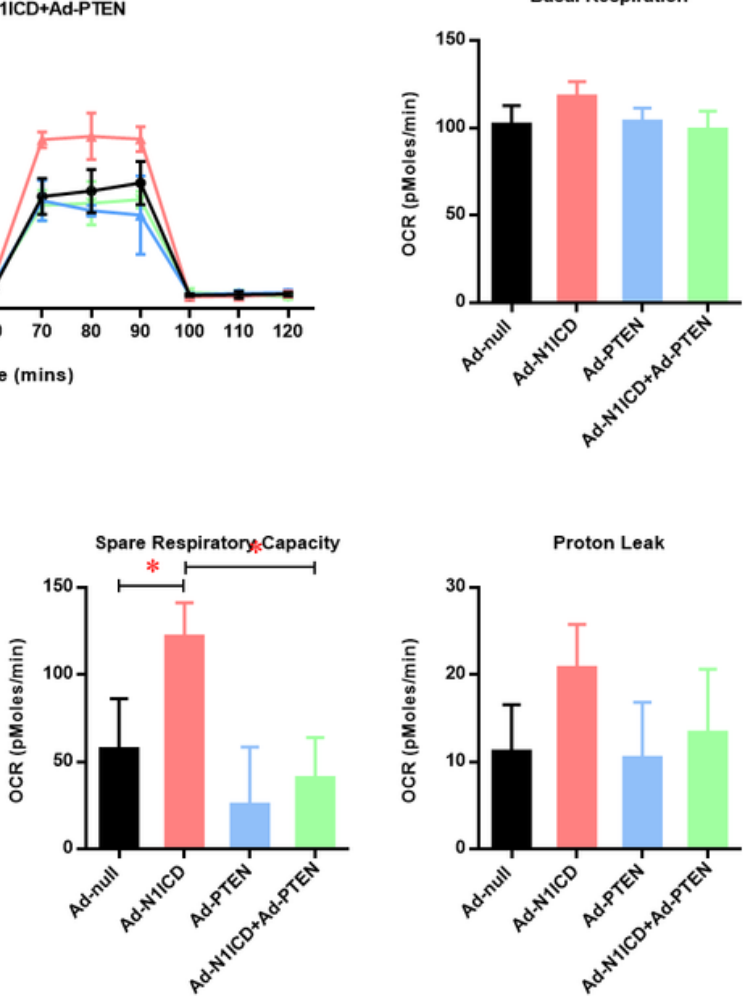

Proton Leak

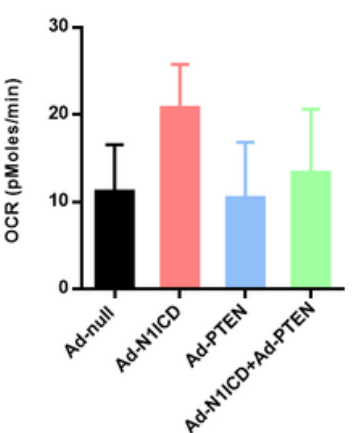

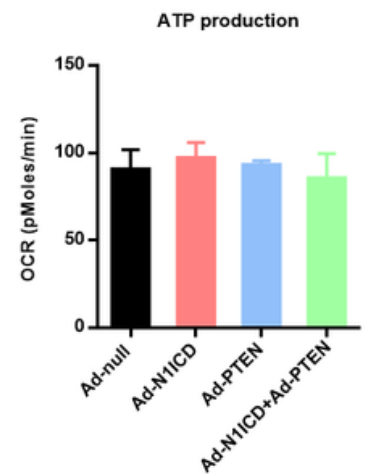

Non-mitochondrial Respiration

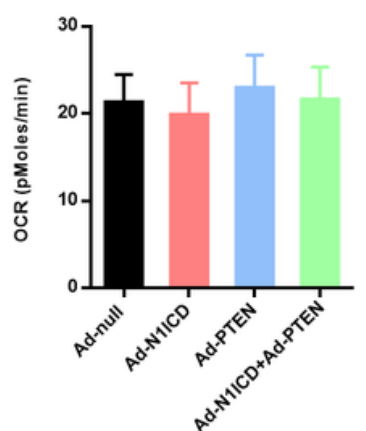

Figure 3

PTEN over-expression impaired the protective effect of N1 ICD against hypoxia/reoxygenation induced mitochondrial dysfunction in neonatal cardiomyocytes. (A) The ATP content and (B) ATP synthase activity was analyzed by quantitative kits; (C) The mitochondrial bioenergetics was measured by the Seahorse XFp Extracellular Flux Analyzer. The basal respiration (BR), ATP production, maximal respiration (MR) and spare respiratory capacity (SPR), Proton Leak and Non-mitochondrial respiration were analyzed $\mathrm{N}=3 ;{ }^{*} P<05$ versus indicated group. 


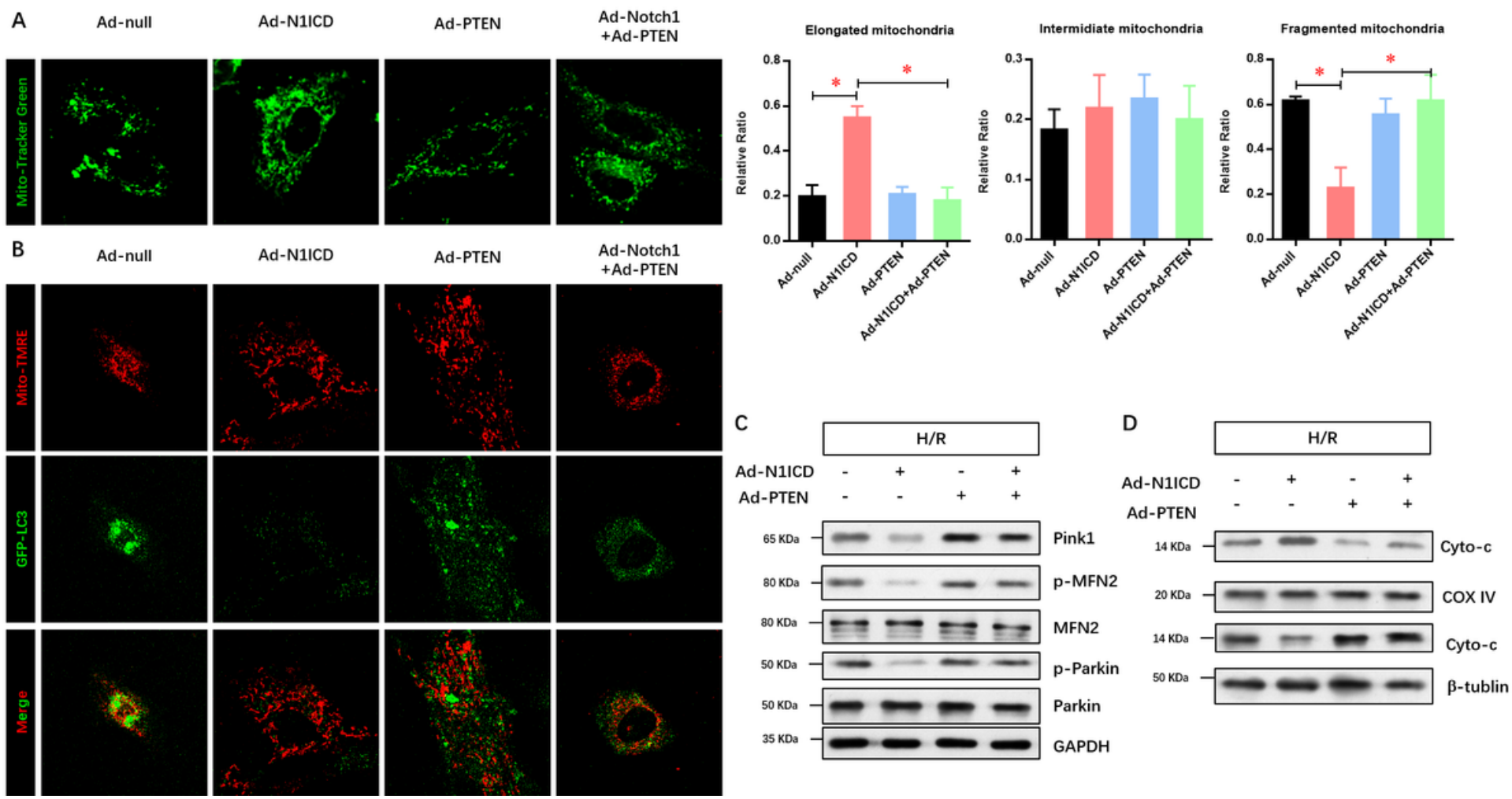

Figure 4

PTEN over-expression impaired the protective effect of N1ICD against hypoxia/reoxygenation induced mitochondrial fragmentation and mitophagy in neonatal cardiomyocytes. (A) Mito-tracker Green staining and confocal microscopy were used to observe the morphology mitochondrial fragmentation in neonatal cardiomyocytes cells; (B) The mitophagy in GC cells was analyzed via the colocalization of GFP-LC3 puncta with Mito-TMRE-stained mitochondria as an index of mitophagy in in neonatal cardiomyocytes cells; (C) The regulatory molecules of Mitochondrial fission and fusion, including Pink1, Parkin1 and MFN2, was analyzed; (D) The regulatory molecule of mitochondrial pathway apoptosis, indicated by Cytochrome c release from mitochondria was analyzed $\mathrm{N}=3 ;{ }^{\star} P<05$ versus indicated group. 
A
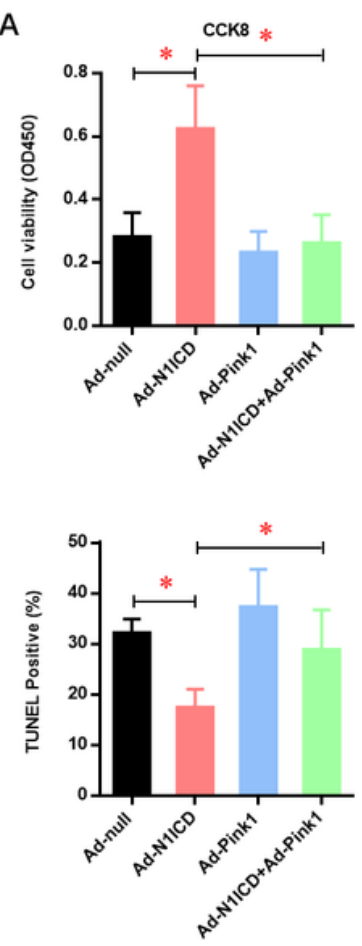

B
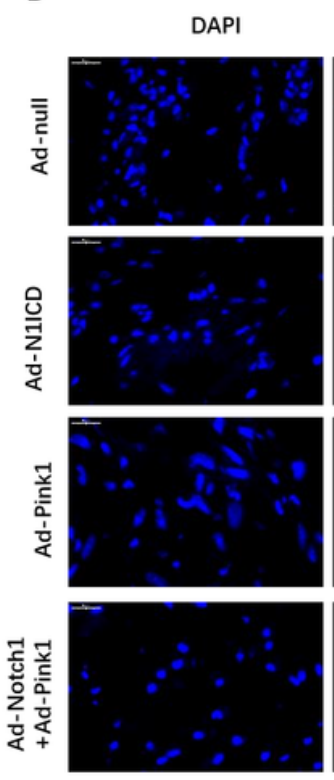

TUNEL
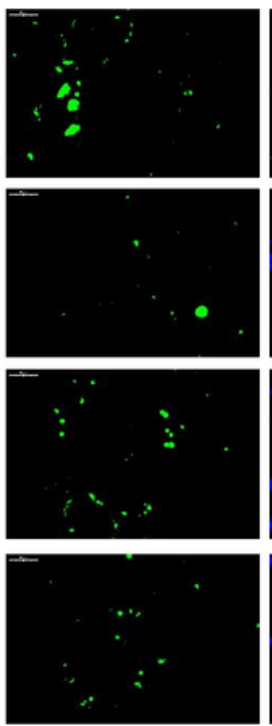
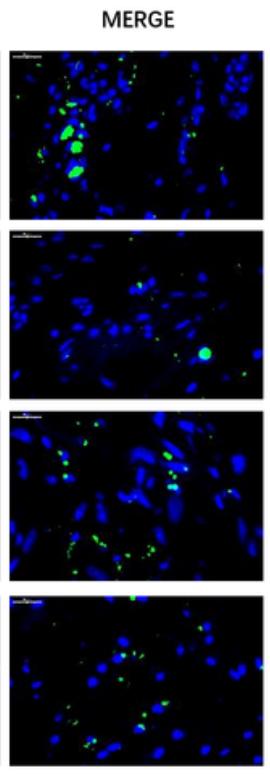

C
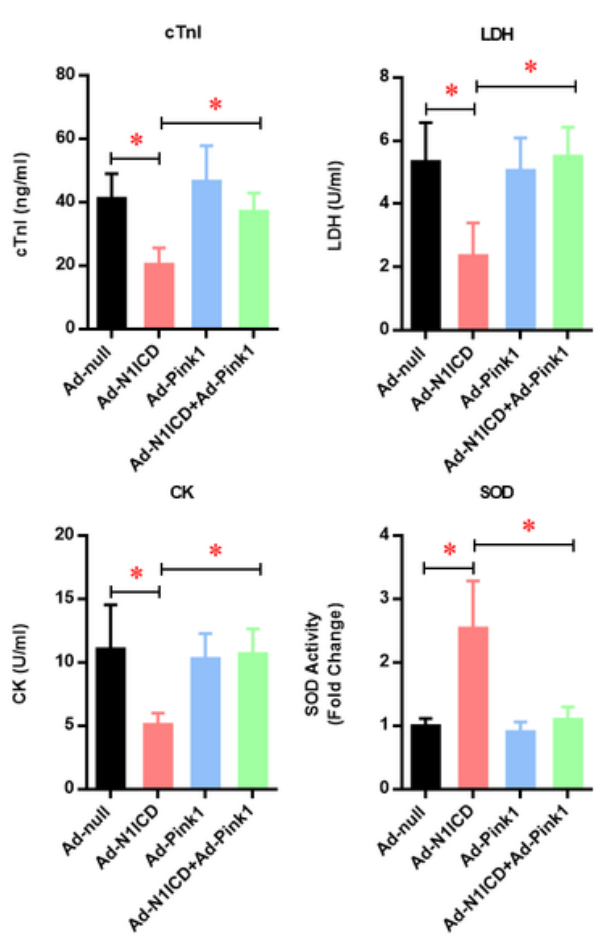

Figure 5

Pink1 over-expression impaired the protective effect of N1ICD against hypoxia/reoxygenation induced injury in neonatal cardiomyocytes. (A) The cellular viability was detected by CCK-8 assay; (B) The percentage of apoptotic neonatal cardiomyocytes was analyzed by TUNE staining; (C) The level of cTnl in the culture medium were also detected at the end of the reperfusion to assess myocardial injury; (D) The activity of LDH and (E) CK were evaluated by Electrochemiluminescence immunoassay; (F) The levels of SOD was analyzed by ELISA Kit; $N=3 ;{ }^{\star} P<05$ versus indicated group. 
A

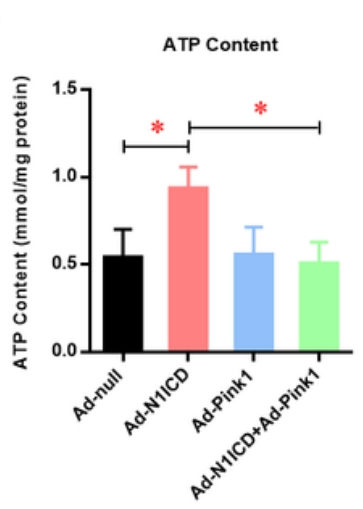

B

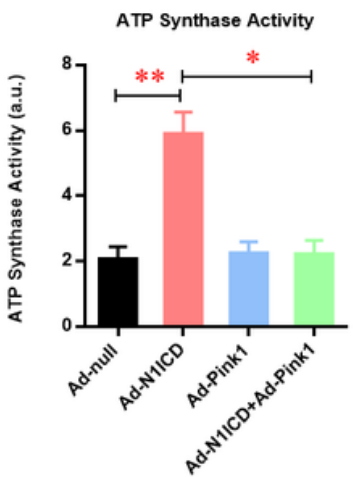

C
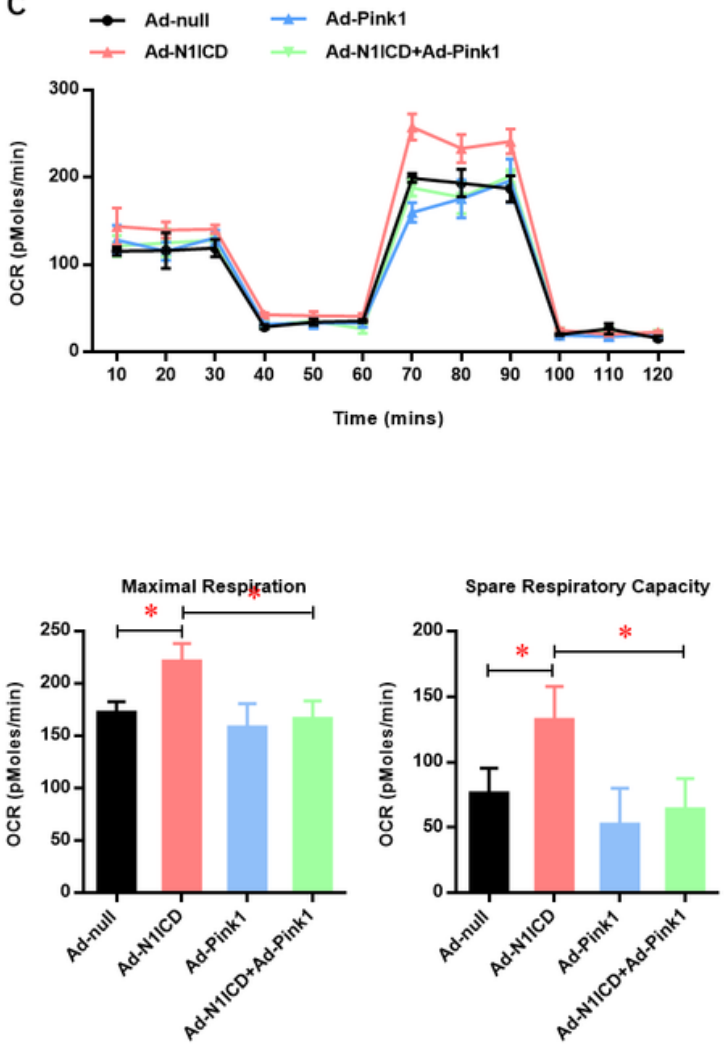

Basal Respiration
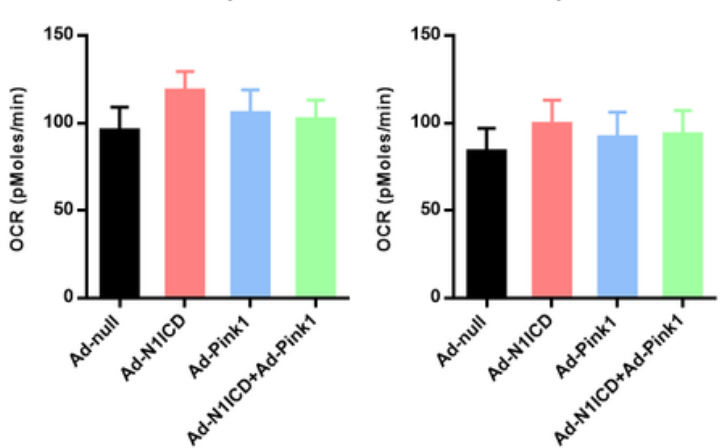

Proton Leak

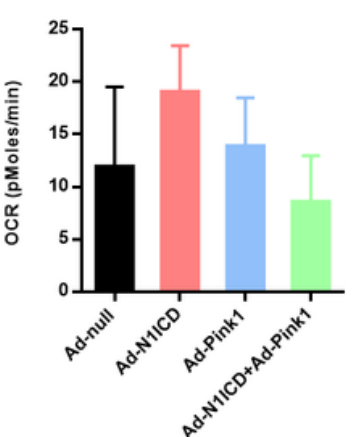

Non-mitochondrial Respiration

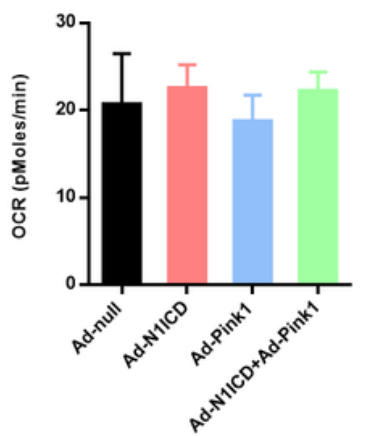

Figure 6

Pink1 over-expression impaired the protective effect of N1 ICD against hypoxia/reoxygenation induced mitochondrial dysfunction in neonatal cardiomyocytes. (A) The ATP content and (B) ATP synthase activity was analyzed by quantitative kits; (C) The mitochondrial bioenergetics was measured by the Seahorse XFp Extracellular Flux Analyzer. The basal respiration (BR), ATP production, maximal respiration (MR) and spare respiratory capacity (SPR), Proton Leak and Non-mitochondrial respiration were analyzed $\mathrm{N}=3 ;{ }^{*} P<05$ versus indicated group.
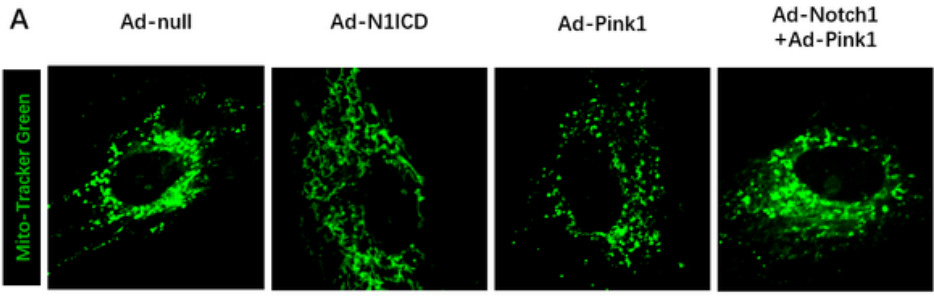

Elongated mitochondria
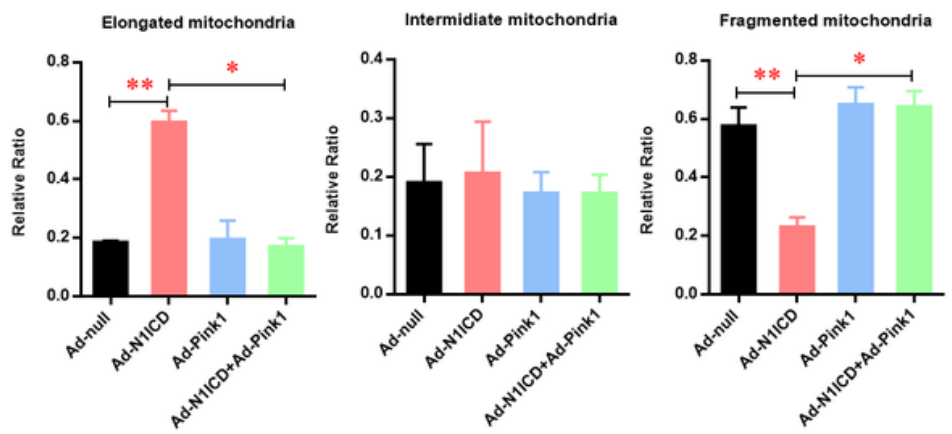
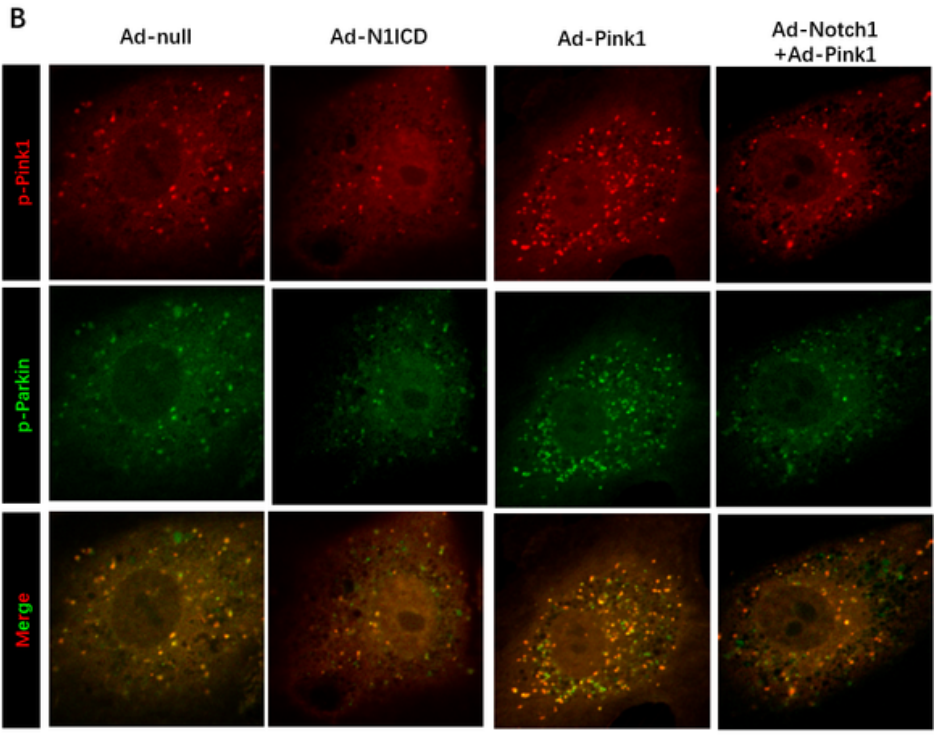


\section{Figure 7}

Pink1 over-expression impaired the protective effect of N1ICD against hypoxia/reoxygenation induced mitochondrial fragmentation and mitophagy in neonatal cardiomyocytes. (A) Mito-tracker Green staining and confocal microscopy were used to observe the morphology mitochondrial fragmentation in neonatal cardiomyocytes cells; (B) The mitophagy in GC cells was analyzed via the colocalization of GFP-LC3 puncta with Mito-TMRE-stained mitochondria as an index of mitophagy in in neonatal cardiomyocytes cells; $\mathrm{N}=3 ; * P<05$ versus indicated group. 
A

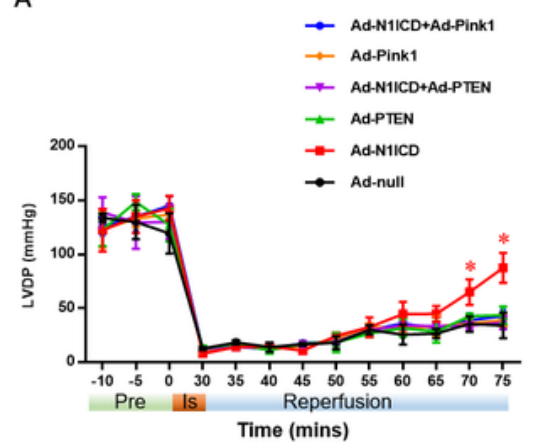

$\rightarrow$ Ad-N11CD+Ad-Pink1

$\rightarrow$ Ad-Pink1

$\rightarrow$ Ad-N1ICD+Ad-PTEN

$\mp$ Ad-PTEN

$\rightarrow$ Ad-N1ICD

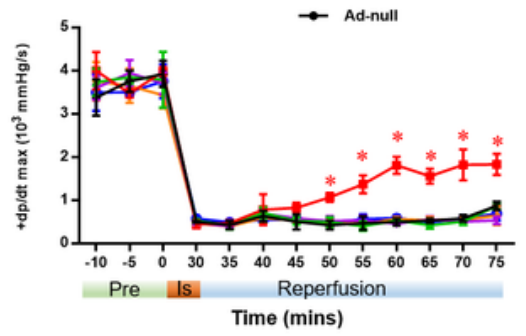

C

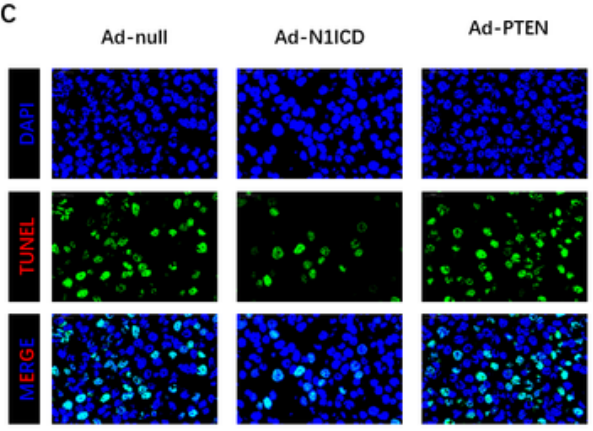

D
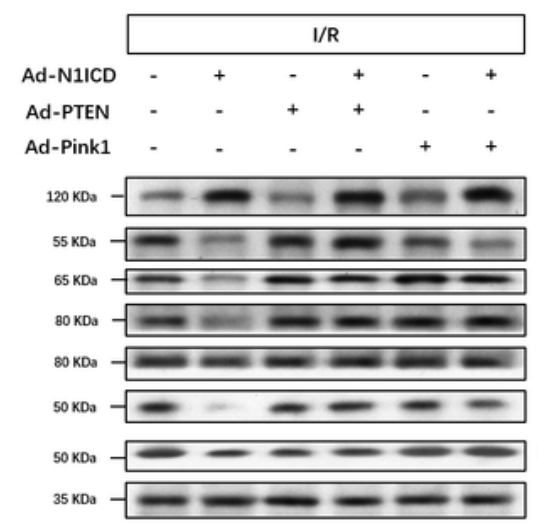

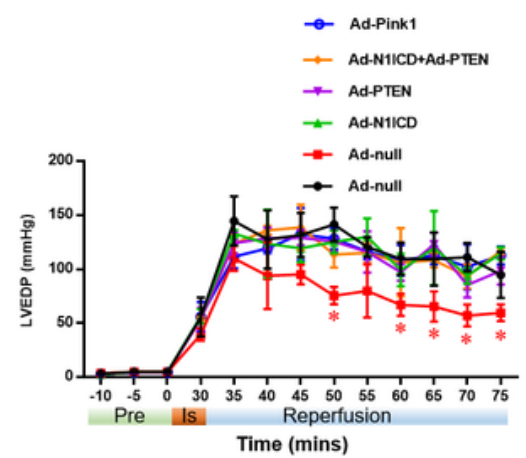

$\rightarrow$ Ad-N11CD+Ad-Pink1

$\rightarrow$ Ad-Pink1

$\rightarrow$ Ad-N1ICD+Ad-PTEN

$\mp$ AdPTEN

$\rightarrow$ Ad-N11CD

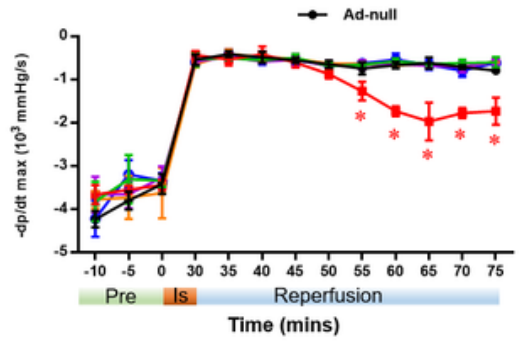

Ad-Notch1
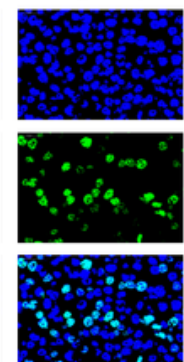

Ad-Pink1
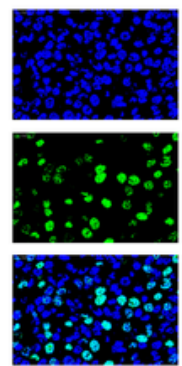

B
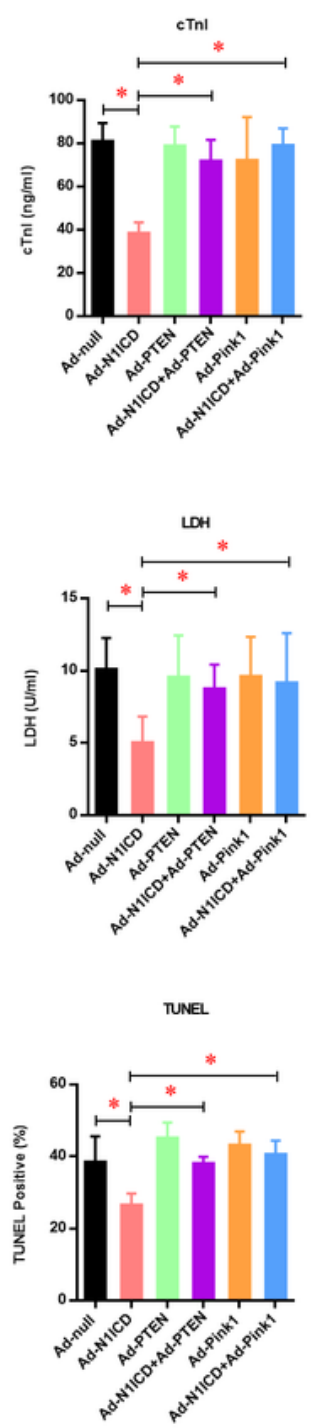

E
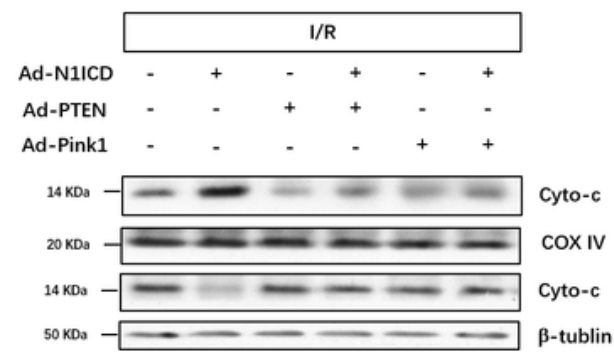

N1ICD

PTEN

Pink1

p-MFN2

MFN2

p-Parkin

GAPDH

Figure 8

\section{N1ICD protective against ischemic reperfusion injury via suppressing PTEN-Pink1 mediated}

mitochondrial dysfunction and mitophagy in vivo. (A), Representative traces and summarized data of LV pressure (LVP) during ischemic-reperfusion (I/R) in isolated rat hearts from indicated groups; (B) The level of cTnl was detected at the end of the reperfusion to assess myocardial injury; (C) The activity of LDH was evaluated by Electrochemiluminescence immunoassay; (D) Representative pictures and quantitative 
results of TUNEL stained cardiac sections were shown; $(E)$ The regulatory molecules of Mitochondrial fission and fusion, including Pink1, Parkin1 and MFN2, was analyzed; (F) The regulatory molecule of mitochondrial pathway apoptosis, indicated by Cytochrome $\mathrm{c}$ release from mitochondria was analyzed $\mathrm{N}=5 ;{ }^{*} P<05$ versus indicated group. 